\title{
RADIATION-HARD BEAM POSITION DETECTOR FOR USE IN THE ACCELERATOR DUMP LINES *
}

\begin{abstract}
P. Degtiarenko, D. Dotson, A. Freyberger, V. Popov, Jefferson Lab, Newport News, VA 23606, USA Abstract

A new method of beam position measurement suitable for monitoring high energy and high power charged particle beams in the vicinity of high power beam dumps is presented. We have found that a plate made of Chemical Vapor Deposition (CVD) Silicon Carbide (SiC) [1] has physical properties that make it suitable for such an application. CVD SiC material is a chemically inert, extremely radiation-hard, thermo-resistive semiconductor ca-

phorescent viewscreen (Refs. [2], [3]) with a video camera readout system. A special optical design was required to bring the image of the viewscreen to a shielded enclosure, in which a camera could survive the radiation. Despite this effort to protect the optical components, cameras typically have a short lifespan in these conditions, and the viewscreens fail after prolonged irradiation. The need for an independent beam position measurement system for the dumpline has brought us to the subject of this work.
\end{abstract} pable of withstanding working temperatures over $1500^{\circ} \mathrm{C}$. It has good thermal conductivity comparable to that of Aluminum, which makes it possible to use it in high-current particle beams. High electrical resistivity of the material, and its semiconductor properties allow characterization of the position of a particle beam crossing such a plate by measuring the balance of electrical currents at the plate ends. The design of a test device, and first results are presented in the report.

\section{INTRODUCTION}

Some of the new fixed target experiments approved for runtime at the Continuous Electron Beam Accelerator Facility (CEBAF) at Jefferson Lab (JLab) require complicated beam steering efforts to keep the electron beam at its nominal position at the face of the beam dumps after it passes through the experimental targets. The new experiments employ magnetic fields in the areas near the target, which are capable of moving the beam away from the dump face and damaging the dumpline equipment with the hundred-kW beams. Therefore, the problem of reliable beam position measurement in the dumpline areas has become more critical.

Precise and reliable position monitoring of high energy and high power accelerated particle beams in the vicinity of high power beam dumps has always presented a technical challenge. The beam quality in those areas is often very poor, and any equipment positioned there must be extremely resistant to radiation damage. Methods involving Radio Frequency (RF) beam time structure measurements often do not work in these areas due to the RF disturbances, and the disturbances in the transverse beam profiles, caused by beam scattering in the irradiated targets. The methods involving moving parts to perform beam profile scans, and optical beam viewers all require high reliability of the control and measurement equipment working in the high radiation environment.

Until recently, the only means to see the beam position at the entrance of the beam dump tunnel at JLab was a phos-

\footnotetext{
* Work supported by the U.S. Department of Energy under contract DE-AC05-84ER40150; U.S. patent pending
}

\section{NEW BEAM POSITION DETECTOR}

The schematic drawing of the device is shown in Fig. 1.

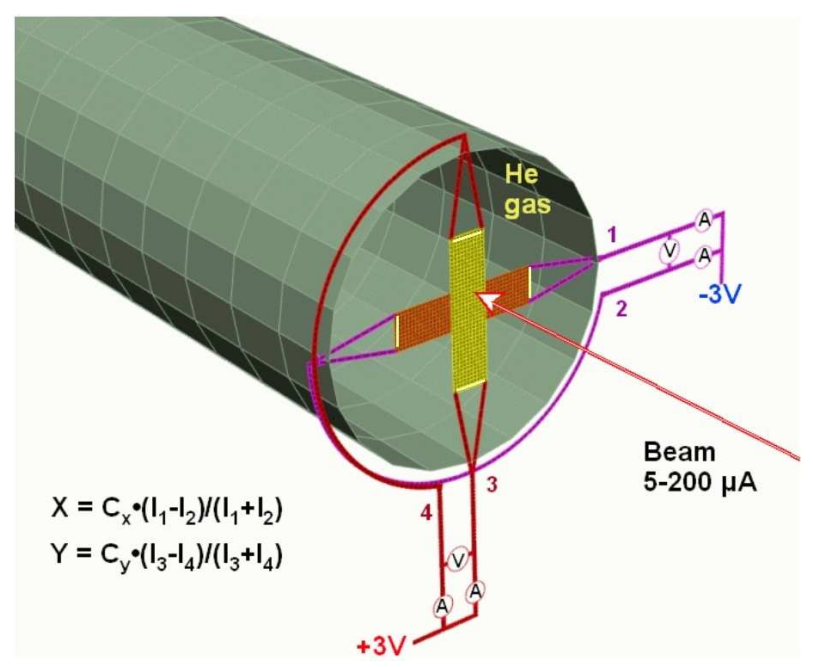

Figure 1: Schematic layout of the BPD.

\section{General Layout and Detector Design}

The Beam Position Detector (BPD) consists of the two thin plates $(200 \times 50 \times 0.25 \mathrm{~mm})$ made of CVD SiC material of standard (high) resistivity; typical electric resistance between plate ends is $200-600 \mathrm{k} \Omega$. One of the plates is suspended horizontally, and the other one vertically in the middle of He gas - filled pipe leading to the CEBAF high power beam dump. The plate width was determined by the need to measure the transverse beam position within the $5 \mathrm{~cm}$ by $5 \mathrm{~cm}$ area, determined by the dimensions of the dump entrance window. The plate thickness was chosen to be as thin as technologically feasible, and the plate length was sized sufficiently large to bring the plate endpoints farther away from the center to avoid the beam damage to the end contacts. The vertical and horizontal plates are parallel to each other and orthogonal to the beam direction. They are separated by $\approx 5 \mathrm{~mm}$ distance along the beam. 
The plates are electrically attached at the ends to the relatively thick $(\approx 1 \mathrm{~mm}$ in diameter) copper wire, serving as a mechanical support, and as an additional heat sink for the plates. Placed in Helium atmosphere, the plates are capable of withstanding the CEBAF operational limit of 200 $\mu \mathrm{A}$ electron beam coming through them. The power deposited in one plate by such beam would be $\approx 30 \mathrm{~W}$, bringing the conservatively estimated equilibrium temperature of the plate at the ends to no more than $150^{\circ} \mathrm{C}$. The temperature at the beam center would strongly depend on the beam size, and should be no larger than $500-800^{\circ} \mathrm{C}$ for the extreme operating conditions. Reliable electric contacts at the plate ends are provided by using conductive paint covered by conductive radiation-hard ceramics. The assembly is set up in an aluminum ring inside the pipe with an opportunity for position adjustment. The copper wires are attached to the electrical feedthroughs in the pipe, and the wire cable is brought to the control box outside of the dump tunnel in a radiation-safe place.

The control box provides low voltage potential difference between the plates, and is capable of measurement of electric DC currents from/to the plate ends. One plate voltage potential is set at +3 Volts relative to the base ground (chassis, cable electric shield, the beam pipe elements), and the other plate potential is set at -3 Volts. The potential difference causes the electric current between the plates when the beam is present, due to significant gas ionization by the beam. The two-plate assembly works similar to a lowvoltage ionization chamber. The sum of currents read from the horizontal plate equals the sum of currents read from the vertical plate with opposite sign. Such current sums are roughly proportional to the beam current intercepted by the sensitive area where the plates are intersecting.

As the plates are resistive, the balance of the currents read off a plate represents beam position information. If an ideal symmetric beam is hitting an ideal plate in the middle, the symmetry requires that the currents read from both ends are equal. The displacement of the beam could be correlated with the asymmetry of the current readings from the two ends. The ideal relation between the coordinate and the currents is as shown in Fig. 1.

The real situation is complicated by the fact that the resistivity of CVD SiC has a very strong temperature dependence, and the heating of the plate by the beam is very non-uniform and dependent on the beam position at the plate. For example, the temperature pattern for the beam hitting the center of the plate is different from the pattern for the beam hitting the edge. Thus the relation between the readout currents and the beam position is non-linear and requires detailed study and calibration. In addition, this temperature sensitivity necessitates waiting until thermal equilibrium is achieved before stable current readings can be obtained. The first experience with beam on the BPD device showed that the characteristic time to reach stable readings is 90-120 seconds after each beam interruption. Once equilibrium is reached, the readings are stable and reproducible, with position resolution of $\approx 1-2 \mathrm{~mm}$.

\section{Calibration Procedure}

Presently, the BPD device is installed at the entrance flange of the He-filled pipe inside the beam dump tunnel in the Hall A at CEBAF. The anodized flourescent screen of the optical viewer is located $\approx 10 \mathrm{~cm}$ upstream of the BPD. Fig. 2 illustrates results of the calibration run in April 2005. Transverse beam coordinates as measured by the BPD during the calibration (red dots) are plotted on top of the reference beam position coordinates obtained from the digitized image of the optical viewer screen (white dots that are mostly covered by the red dots). The coordinates are

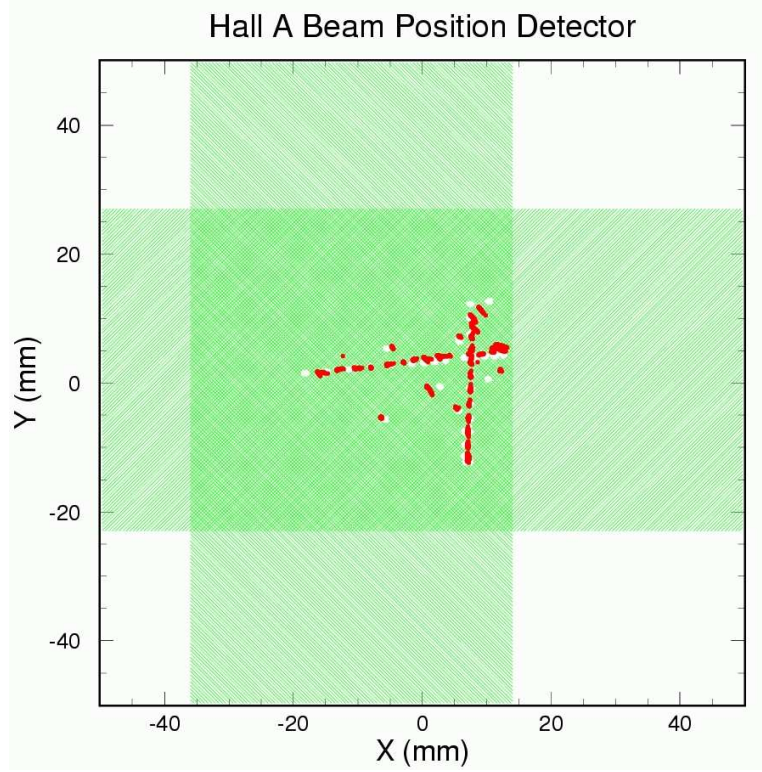

Figure 2: Transverse beam coordinates measured during the calibration. See detailed explanation in the text.

given in the viewer reference frame; the green hatched areas show estimated positions of the vertical and horizontal BPD plates in the viewer frame.

The position of $40 \mu \mathrm{A}, 4.7 \mathrm{GeV}$ electron beam, incident at the BPD, was monitored by the viewer screen; the digitized information on the beam position, and measured current readings from the BPD were recorded once a second by the data acquisition system. The beam steering by the control magnets in the beam line allowed scanning of the sensitive area within a range of $\approx \pm 1 \mathrm{~cm}$ in $\mathrm{X}$ and $\mathrm{Y}$. The correlated readings were used to find the non-linear relations between the BPD current readings and the beam coordinates and subsequently use this information to extract beam coordinate values from the BPD data. Comparison of the viewer and the BPD coordinates after such conversion shows agreement within 1-3 $\mathrm{mm}$ in $\mathrm{X}$ and $\mathrm{Y}$.

\section{Operation Experience}

Fig. 3 illustrates typical operation of the BPD device. X and $Y$ coordinates are measured every second, and plotted against time. All periods with stable coordinate readings 


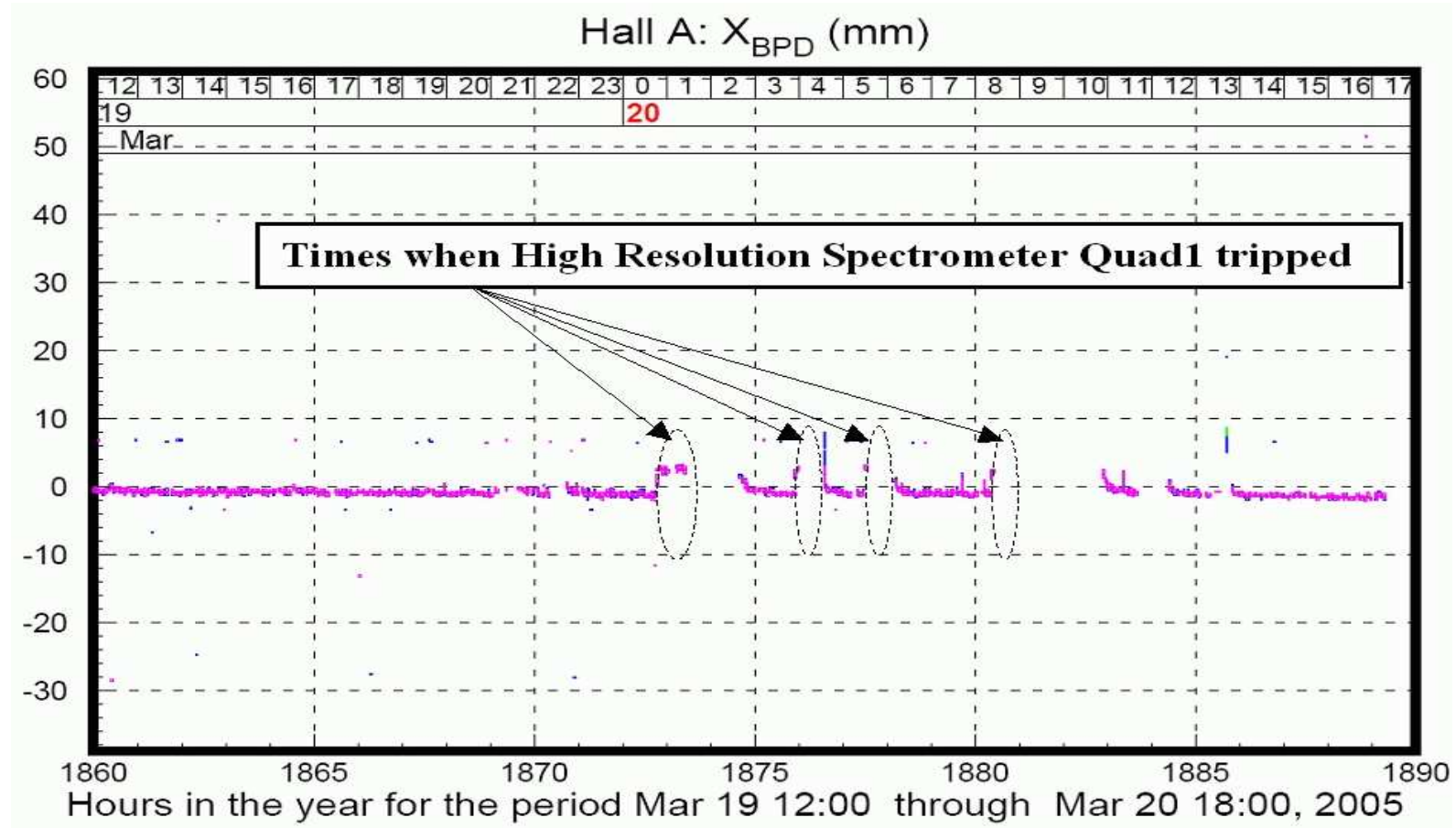

Figure 4: Horizontal beam position plotted against time, measured during March 19-20, 2005 experimental run. Otherwise stable run conditions were interrupted by several trips of a quadrupole magnet in one of Hall A spectrometers in the early morning hours on March 20th. Changes in the magnetic field along the beam path between the experimental target and beam dump tunnel entrance caused horizontal beam position shift of about $4 \mathrm{~mm}$.

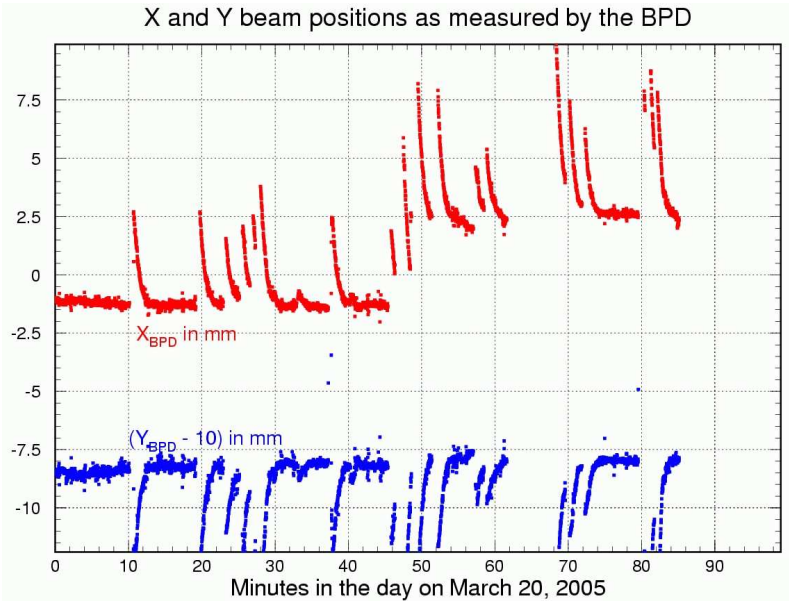

Figure 3: $\mathrm{X}$ and $\mathrm{Y}$ coordinates measured by the BPD during the experimental run. $\mathrm{Y}$ is shifted down by $10 \mathrm{~mm}$ to avoid interference.

correspond to the stable $40 \mu \mathrm{A}, 4.7 \mathrm{GeV}$ electron beam crossing the device. The interruptions in the beam correspond to the interruptions in the readings, followed by the characteristic pattern of recovery, delayed by $90-120 \mathrm{sec}$. The change in X coordinate at around 00:50 is real beam position shift caused by tripping of one of the spectrometer magnets in the Hall not far from the beam line. More extended pattern of $\mathrm{X}$-coordinate readings, covering a period of $\approx 30$ hours of relatively stable operations, is shown in Fig. 4. The first 120 seconds after the end of each beam interruption are not shown in order to clean up the plot. We see a very stable beam delivery during this period, interrupted by several magnet trips that caused shifts in $\mathrm{X}$.

\section{CONCLUSION}

In conclusion, we have demonstrated operation of a new Beam Position Detector device for use in the accelerator dump lines. The first BPDs have survived many hundreds of hours of continuous operation at beam currents up to $100 \mu \mathrm{A}$, logging data for more than a year. The stability and reproducibility of the BPD readings allows calibration to within a few mm in position accuracy. We plan to switch from a passive use of the BPD, as a backup to the present beam viewers, to more critical missions for upcoming experiments.

\section{REFERENCES}

[1] CVD SILICON CARBIDE ${ }^{\circledR}$ is a registered trademark of Advanced Materials, Rohm and Haas Company, http://www.cvdmaterials.com

[2] A. Silva, "Requirements of the CEBAF beam viewer system”, CEBAF technical document CEBAF-TN-93-035, 1993.

[3] A. Freyberger, CEBAF internal communication. 